Discrete Comput Geom 30:165-166

DOI: $10.1007 /$ s00454-003-0444-0

\title{
Guest Editors' Foreword
}

This special issue of Discrete \& Computational Geometry is devoted to the proceedings of two joint U.S.-Hungarian workshops in discrete geometry and convexity: the first in Budapest (July 12-16, 1999), and the second in Auburn, Alabama (March 21-26, 2000). The workshops were supported, in part, by an NSF grant obtained by the U.S. organizers. The Hungarian partners obtained similar support from the Hungarian Academy of Sciences.

The topics of the workshops varied throughout discrete geometry and convexity, and included geometric graph theory, finite point configurations, packing and covering, isoperimetric problems, geometric algorithms, and the theory of polytopes. Both classical and computational aspects of the field were well represented.

The spirit of collaboration permeated the workshops, inspiring the writing, already during the time between the workshops, of two joint publications included in this issue: one by J. Pach, J. Solymosi, and G. Tóth, and the other by I. Bárány, K. Kuperberg, and T. Zamfirescu. Thanks to the increase of personal contacts between the participants from the two countries, the collaboration is expected to continue in years to come and to result in many more joint articles.

The issue consists of 15 articles whose topics well represent those of the two workshops. The centerpiece of the collection is the breakthrough result on Straightening Polygonal Arcs by R. Connelly, E. Demaine, and G. Rote. The three co-authors solve in an elegant manner an intuitively appealing, old problem: can every planar polygonal arc with rigid edges and flexible joints be continuously transformed into a straight (collinear) arc, while remaining a topological arc during the entire transformation? Some basic ideas of rigidity theory - the area of expertise of the first author-are used extensively in this joint paper that answers the question in the affirmative. The legacy of the late Paul Erdôs can be seen in four of the articles included that address his problems and represent his style in combinatorial geometry, authored by G. Károlyi and P. Valtr, by J. Pach, J. Solymosi, and G. Tóth, by J. Solymosi, and by A. Pór. In the theory of polytopes, articles by I. Bárány, K. Kuperberg, and T. Zamfirescu and by K. Böröczky and B. Csikós present surprising examples, and the article by I. Talata derives an algebraic formula for the volume of the medial section of the $n$-dimensional simplex. Density problems are treated in the articles written by K. Böröczky, Jr. and by A. Heppes, while articles by 
A. Bezdek and by D. Ismailescu address geometric extremum problems in the classical theory of packing and covering. A further three areas of geometry are represented by contributions in the articles: by K. Bezdek and A. Blokhuis, to the theory of integer lattices; by J. Kincses, to geometric tomography; and by S. Langerman and W. Steiger, to computational geometry.

The guest editors wish to express their gratitude to all the authors for their valuable contributions to this special issue.

Gábor Fejes Tóth

Włodimierz Kuperberg 